\title{
Development of the substitution lines of bread wheat with introgressed pubescence from T. timopheevii and their study in contrasting irrigation conditions
}

\author{
Simonov A.V. ${ }^{*}$, Osipova S.V. ${ }^{2}$, Permyakov A.V. ${ }^{2}$, Permyakova M.D. ${ }^{2}$, \\ Kovaleva N.M. ${ }^{1}$, Chistyakova A.K. ${ }^{1}$, Pshenichnikova T.A. ${ }^{1}$ \\ ${ }^{1}$ Institute of Cytology and Genetics, SB RAS, Novosibirsk, Russia \\ ${ }^{2}$ Siberian Institute of Plant Physiology and Biochemistry, SB RAS, Irkutsk, Russia \\ *e-mail: sialexander@bionet.nsc.ru
}

The leaf pubescence serves as a mechanism of adaptation to adverse environmental conditions by forming a near-surface protective layer that delays air currents and scatters solar radiation. In bread wheat, the trait is controlled by dominant genes in chromosomes 4B and 7B. T. timopheevii species has rare but very long pubescence. The introgressive line 821 carries this new type of pubescence and contains the fragment from T. timopheevii on $5 \mathrm{~A}$ chromosome marked with the Xgwm 179 microsatellite marker. This pubescence was introduced into the single chromosome substitution lines for $5 \mathrm{~A}$ chromosome of two cultivars, Saratovskaya 29 (S29) and Diamant 2 (Dm2), contrasting for leaf pubescence and drought tolerance. The effect of this introgression on a number of physiological parameters has been established. In the line Dm2(821 5A), the stomatal conductance and transpiration rate decreased by 2.5-4 times under normal irrigation and drought, the photosynthetic activity decreased by about a quarter, and the water use efficiency (WUE) doubled in comparison with the poorly haired recipient. This introgression practically did not affect the physiology of S29 under normal watering. However, under drought conditions, S29(821 5A) more than doubled the stomatal conductance and the rate of transpiration. The WUE more than doubled, while the photosynthetic activity remained at the same level. The productivity parameters of the line S29(821 5A) did not differ significantly from the recipient under normal watering. The line Dm2(821 5A) surpassed the recipient by one and a half times for the weight of grain and the number of grains from the plant. Under drought, the plants of both substitution lines switched to a moisture saving mode, and reduced the productivity more than the recipients did. Chromosome substitution increased the raw gluten content in grain by a quarter in S29 and to some extent in Dm2.

Acknowledgements: This study was funded by RFBR grant No. 18-04-00481. 